\title{
Criminologie
}

\section{Femme battue et mari « batteur » : une reconstruction médiatique dans La Presse au XIXe siècle}

\section{Joane Martel}

Volume 27, numéro 1, 1994

Analyse spatiale du crime

URI : https://id.erudit.org/iderudit/017351ar

DOI : https://doi.org/10.7202/017351ar

Aller au sommaire du numéro

\section{Éditeur(s)}

Les Presses de l'Université de Montréal

ISSN

0316-0041 (imprimé)

1492-1367 (numérique)

Découvrir la revue

Citer cet article

Martel, J. (1994). Femme battue et mari « batteur " : une reconstruction médiatique dans La Presse au XIXe siècle. Criminologie, 27(1), 117-134. https://doi.org/10.7202/017351ar

\section{Résumé de l'article}

We would assume that mass newspaperdom which is slowly introduced by the end of the XlXth century was tributary to the dominant conception of liberties which attributed rights to the householder and garanteed the privacy of the home. The evocation of these rights would then give rise to hesitations to intervene publicly in family quarrels. But, a documentary research of the Quebec daily La Presse reveals, on the contrary, that it proceeded to expose virulently cases of domestic violence in such a way that husbands became the main target of sarcasm. Therefore, unlike the ideological positions generally conceded to the Victorian project, the general tendancy to reduce the Victorian moralism to two main angles - being the purification of sexual behaviors and the promotion of a holy, asexual and family image of women - is questioned. The social project of the XlXth century appears more complex than what is generally thought since it proceeds not only to subject women but looks concurrently to "civilize" and moral-he the conduct of men. 
We n'ould assume that mass new'spaperdom which is slowly introduced by the end of the XIXth century was tributary to the dominant conception of liberties which attributed rights to the houscholder and garanted the privacy of the home. The evocation of these rights would then give rise to hesitations to intervene publicly in family quarrels. But, a documentary research of the Qucbec daily La Presse reveals, on the contrary, that it proceeded to expose virulently cases of domestic violence in such a way that husbands became the main target of sarcasm. Therefore, unlike the ideological positions generally conceded to the victorian project, the general iendancy to reduce the victorian moralism to two main angles - being the purification of sexual behaviors and the promotion of a holy, asexual and family image of women - is questioned. The social project of the XIXth century appears more complex than what is generally thought since it proceeds not only to subject women but looks concurrenly to "civilize" and moral. ize the conduct of men.

Le crime en tant que fabrication juridico-pénale est repris et reconstruit de diverses façons, entre autres par les institutions de contrôle social parmi lesquelles se distinguent l'appareil de justice criminelle, le droit et les médias (Curran, 1978). Par le jeu de leurs pratiques de rétention, d'inflation des signes, de redondance des mots et des idées, en somme de rhétorique de persuasion, ces derniers parviennent, en effet, à imposer des représentations particulières du crime. Leur couverture médiatique sert alors essentiellement à réduire le caractère équivoque du monde et à neutraliser la menace qu'il comporte pour l'image du consensus social pour, ensuite, reproduire cette image de l'ordre. C'est en tant que telle, que la construction journalistique de l' «événement " nous a incité à questionner la représentation de la victimisation et de la marginalisation que véhiculent les médias. À cet effet, le présent travail a cherché plus spécialement à retracer l'image des femmes victimes de violence au foyer telle qu'elle est présentée au travers de la criminalité rapportée à la fin du siècle passé par la presse québécoise pour, ensuite, voir comment cette image s'articule au contexte

1. Chercheure, Groupe de recherche el d'analyse sur les politiques et les pratiques pénales (GRAPP), Département de sociologie. Université du Québec à Montréal. C.P. 8888 , Montréal H3C 3 P8. 
socio-politique de l'époque ${ }^{2}$. À cet égard, nous questionnerons la tendance générale à réduire le moralisme victorien à deux aspects soit l'assainissement des comportements sexuels et la promotion d'une image des femmes qui se veut sainte, asexuée et familiale. Le projet victorien est. en fait, beaucoup plus complexe et nous verrons qu'il ne s'attarde pas uniquement à l'assujettissement des femmes.

Notre objectif méthodologique principal étant de faire ressortir les caractéristiques des récits de crimes en vue de saisir l'image des femmes qui y est véhiculée, une recherche documentaire et une étude de presse de ce type particulier de nouvelles ont donc été entamées. Au cours de la recherche initiale ${ }^{3}$, l'analyse d'un tel corpus avait permis de catégoriser les délits que rapporte la presse écrite, et dont les femmes étaient victimes à la fïn du XIX $\mathrm{X}^{\mathrm{e}}$ siècle, en deux sections globales, soit les délits à caractère sexuel et la violence domestique. À l'intérieur des premiers se construit une image des femmes victimes qui est traitée différemment de celle présentée lors de crimes de violence au foyer; chacun de ces deux groupes d'infractions constituant alors en lui-même un sujet de recherche distinct. C'est sur ce second type de situations et d'images de presse que porte le présent article. Dans le but de demeurer Fidèle au corpus, J'exposé qui suivra ne fera pas usage du concept de violence conjugale car celui-ci n'a pas été recensé dans les récits journalistiques analysés. Nous préconiserons plutôt les notions de «violence domestique » et de «violence au foyer», telles que les véhiculait la presse québécoise de l'époque.

Parmi l'ensemble des quotidiens québécois existant vers la fin du siècle dernier, un seul a retenu notre attention comme source primaire de donnés, soit le journal La Presse. Celui-ci est mis sur pied à une époque où, sous l'inéluctable pression d'un modernisme fougueux, Je journalisme populaire illustré et «la presse à un sou» commencent sérieusement à détrôner la presse d'idées au Québec (Beaulieu et Hamelin, 1977), La

2. Cet article est extrait d'une recherche qui s'est intéressée plus globalement à l'évolution de la représentation journalistique des contrevenant es et des femmes victimes de crimes dans le quotidien La Presse sur une période d'un siècle, plus précisément de la fin du XIX ${ }^{\mathfrak{e}}$ siècle à la fi du XXe siècle. Deux blocs historiques avaient alors été mis en parallèle (1886-1889 et 1986-1989). Une analyse complémentaire dans des périodes intermittentes se situant à intervalles réguliers entre 1886 et 1989 avait été adjointe à ce dépouillement initial. Les données dont fait état cet article retracent non seulement la représentation journalistique de la violence domestique dans les années 1886-1889 mais remontent à la création de La Presse en 1884.

3. Voir Martel, J., La reconstruction de la criminalité à travers La Presse (18861989) : l'image de la contrevenante et de la femme victime, Université d'Ottawa, 1991, ronéo. Nous tenons à remercier $M$. André Cellard pour ses commentaires judicieux, ainsi que le Ministère des Collèges et des Universités de l'Ontario pour son soutien financier. 
Presse et ses semblables exhortent alors les journaux d'opinions à se convertir aux exigences de ce nouveau type de journalisme qui approche le monde sous un angle différent. En effet, à l'opposé des entreprises de presse militante plus anciennes, le journalisme populaire est plutôt dirigé vers un lecteur moins engagé politiquement, un public fervent d'informations et d'images qui préfère un compte rendu succinct de nouvelles rédigées dans un style vif ainsi que des éditoriaux opiniâtres (Felteau, 1983 : Voumvakis et Ericson, 1984). Le dernier cinquième du $\mathrm{XIX}^{\mathrm{e}}$ siècle verra alors éclore ce type de presse à bon marché orienté vers les masses et cherchant à rejoindre un large éventail de consommateurs dans diverses couches de la population, s'adressant ainsi à l'ensemble et non à une élite particulière. La Presse annoncera, à cet effet. son caractère «conservateur de principes» mais s'estimera "parfaitement indépendant et libre de tout contrôle officiel» et de tout esprit partisan (Felteau. 1983, p. 59). Ce dernier aspect de la presse populaire s'avère important pour notre étude puisque celle-ci ne tente pas d'analyser la représentation élitiste des femmes que peuvent véhiculer certains quotidiens militants ou certains autres dits « de qualité ». Elle cherche plutôt à reconstruire la représentation qui est diffusée à la population en général et qui risque, par conséquent, d'influencer les mentalités de l'époque dans la mesure où elle participe à la problématisation sociale de certains comportements.

Par ailleurs, le choix méthodologique du journal La Presse s'explique également par un certain nombre d'autres raisons. Cette nouvelle presse, qui s'implante donc dans les années 1880 , est également différente de la presse militante en ceci qu'elle rapporte plus systématiquement les affaires criminelles. Mais, malgré sa couverture substantielle du crime, La Presse demeure, à l'époque, un quotidien de bonne qualité, accordant $50 \%$ de son espace de rédaction aux informations ayant trait à l'économie ou à la scène locale (Rutherford, 1982). De plus, les allures populistes du journal lui confèrent, dès sa création en 1884, un immense succès qui se traduit par un taux de circulation qui surpasse, en moins de cinq ans, celui des deux plus grands quotidiens francophones du pays : Le Monde et L'Électeur (Rutherford, 1982). La Presse se dissémine donc rapidement et ce, jusqu'à l'intérieur des foyers des milieux ouvriers qui ne s'étaient même jamais abonnés à un journal auparavant. Il devient ainsi, à la fin du siècle dernier, le quotidien francophone le plus important parmi les journaux populaires. Par conséquent, ses constructions risquent fort d'être exemplaires du type de société dans laquelle il évoluait. Néanmoins, soulignons que notre corpus ne rend pas compte de la vie des Québécoises et des Québécois à la fin du $\mathrm{XIX}^{\mathrm{e}}$ siècle, mais il permet, à tout le moins, de cerner certains des discours prescriptifs qui leur étaient adressés ; discours dont l'importance même leur est conférée par la diffusion à grande échelle de La Presse partout au Québec. Retracer ainsi les représentations que se font les 
journalistes des affaires de voies de fait entre conjoints permettra, enfin. de mettre en relief la contribution de la presse à grand tirage à la construction de la violence domestique comme problème social sérieux à l'époque.

Tous les récits de crimes se rapportant à des femmes victimes auraient pu être retenus pour l'analyse, cependant, seuls certains d'entre eux correspondaient à nos exigences méthodologiques. Deux types de comptes rendus se distinguent, en fait, dans la couverture journalistique du crime : les récits primaires et secondaires (Voumvakis et Ericson, 1984). Tandis que les premiers se limitent gênéralement à la description d'incidents criminels, les seconds se composent des réponses apportées aux questions implicitement soulevées dans les récits primaires. On englobe, entre autres, à l'intérieur des récits secondaires les éditoriaux et les dossiers ayant trait à un problème social général qu'amplifie souvent la commission d'un crime (sécurité des lieux, maladies «vénériennes», et). Ces derniers n'ont pas été recensés dans notre étude et ce, principalement à cause de leur nombre relativement faible à l'époque. Seuls les récits primaires ont donc été analysés puisqu'ils comprennent généralement les comptes rendus de crimes (e.g. l'incident lui-même, les suivis, les enquêtes policières, les démarches et les demandes de divers organismes concernés) de même que ceux des tribunaux (cautionnement, procès, sentence $)^{4}$.

Précisons qu'à l'intérieur d'une démarche qualitative, la représentativité du corpus se réfère à la saturation de celui-ci. La clôture du corpus s'effectue lorsque les données recueillies deviennent redondantes. Cette tendance du discours à se refermer sur lui-même découle de l'épuisement du registre des significations et c'est donc dire que l'existence d'une logique particulière a été dégagée du discours journalistique. Or, la saturation des fonctions de celui-ci a rendu l'opération de dépouillement exhaustif des quotidiens superflue.

Les récits retenus pour l'étude ont, par la suite, été analysés de façon à y dégager certaines caractéristiques telles que le type de délit commis. l'attribution du blâme (s'il y a lieu), l'âge et le statut social des femmes impliquées, la présence de connotations sarcastiques à leur égard (e.g. moquerie, raillerie ou ironie insultante ou méchante) ou encore l'usage de métaphores qui. elles, constituent un des véhicules importants par lesquels les médias donnent forme à la défiance pour leur auditoire (Ericson, 1987). Dans le cadre d'une recherche portant sur les nouvelles de crimes, il n'est pas possible de contrôler la diversification interne de l'échantillon (e.g. âge, statut social, ethnie, et.) préalablement à la collecte des données. Ainsi devient-elle une opération méthodologique moins pertinente à notre étude. En outre, il est admis que cette situation pose une certaine limite à la

4. Notons que le processus de collecte des données de la recherche initiale a nécessité le dépouillement de 1554 numéros du quotidien La Presse. 
représentativité du corpus, mais le travail inductif que nécessite. dans ce cas-ci, l'étude des reconstructions médiatiques ne permet pas de contourner cette barrière. Il a, néanmoins, été possible d'attester à posteriori l'existence d'une diversification fortuite dans les nouvelles recueillies et analysées, ce qui a eu pour effet de restreindre l'ampleur de la limite sus-mentionnée.

La partie proprement analytique du texte se divise en deux sections principales. La première résume les éléments du contexte socio-politique - propre au Québec dans la deuxième moitié du XIXe siècle - qui suscitèrent notre intérêt pour cette période historique tandis que la suivante retrace le traitement journalistique particulier dont firent l'objet les femmes victimes de violence domestique.

\section{I - LA FIN DU XIX SIECCLE ET LA PRÉDOMINANCE IDÉOLOGIQUE DU MORALISME VICTORIEN}

La deuxième moitié du XIX ${ }^{\mathbf{e}}$ siècle est demeurée longtemps une période mal connue et peu étudiée en ce qui a trait au traitement que la sociêté québécoise donnait à la violence en ménage. Outre l'existence d'une littérature internationale non négligeable sur le problème de la violence domestique au $\mathrm{XIX}^{\mathrm{e}}$ siècle, l'étude du phénomène, dans le Canada et le Québec de l'époque, ne semble avoir attiré l'attention de la communauté scientifique que très récemment. Ce n'est, en effet, que depuis la fin des années 1980, que le sujet suscite plus d'intérêt de la part des historiennes québécoises et canadiennes (Harvey, 1990 et 1991 ; Backhouse. 1991 : Bradbury, 1993). Pourtant. la fin du siècle passé est une période de changements importants non seulement dans la conjoncture socioéconomique, mais également dans la situation des femmes. À cette époque, les femmes ne participent toujours pas a la vie politique publique et ce, en partie parce que la Chambre s'acharne à ne pas reconnaître les droits du «sexe faible». Mais en 1849 leur position se détériore davantage lorsqu'elles perdent leur droit de vote au palier fédéral après que la question ait antérieurement soulevé des débats en Chambre, en 1828 ainsi qu'en 1834 (Dumont-Johnson, 1971). Quelques années plus tard, la codification des règles issues de lá Coutume de Paris, en 1866, consacrera désormais et pour longtemps l'incapacité juridique des femmes mariées au Québec. Il leur sera interdit, entre autres, de tester, d'entamer des procédures judiciaires ou de démarrer une entreprise ou un petit commerce sans la permission préalable de l'époux (Backhouse, 1991). L'avènement du Code civil viendra donc assurer davantage les assises patriarcales des systèmes juridique et social québécois et consacrer les iniquités de la distribution du pouvoir entre époux et épouse. 
Par ailleurs, un des phénomènes marquants de la deuxième moitié du $\mathrm{XIX}^{\mathrm{e}}$ siècle est assurément l'émergence de la mère-travailleuse canadiennefrançaise. Depuis les années 1850 , et surtout à la faveur de la crise économique du début des années 1870 , il devient de plus en plus fréquent pour les femmes des milieux ouvriers francophones de travailler à l'extérieur du foyer (Cross, 1973). Toutefois, la majorité des femmes qui occupent un emploi rémunéré le font par nécessité. La monoparentalité étant un phénomène urbain important au XIX ${ }^{\mathrm{a}}$ siècle à Montréal, les femmes se retrouvaient ainsi souvent seules après une « séparation de corps $~^{5}$ ou encore la désertion ou la mort du mari. Il devenait alors impératif pour ces femmes de suppléer d'une façon ou d'une autre au salaire de l'époux pour ainsi garantir leur survie et celle de leurs jeunes enfants. Outre l'aide ponctuelle que les voisins et la famille élargie pouvaient dispenser, les femmes seules devaient élaborer tout un arsenal de stratégies visant à supporter leur famille telles que le troc. l'ouverture de petits commerces, l'hébergement de chambreurs ou la gestion de maisons closes. Mais, la plupart des femmes seules de l'époque ne pouvaient pas compter sur un quelconque héritage, sur une pension ou encore sur une assurance-vie leur permettant de démarrer ces petites entreprises. Alors, pour la majorité d'entre elles le travail salarié comportait les meilleures chances d'assurer leur survie, du moins jusqu'à ce que les enfants soient en âge de travailler (Bradbury, 1993).

À l'instar des femmes seules, une certaine proportion de femmes mariées se retrouvèrent également sur le marché du travail dans cette période. Cette situation s'explique principalement par le bas niveau des salaires dans un grand nombre de secteurs industriels et par l'instabilité de l'emploi qui ont engendré une insuffisance des revenus des hommes et nécessitèrent donc de suppléer au manque à gagner à l'aide de salaires d'appoint ; celui de l'épouse, certainement, mais aussi, et surtout, celui des enfants (Bradbury, 1993). La conception de leur revenu comme étant complémentaire à celui du chef de famille justifiera le maintien à un niveau inférieur à celui des hommes, ce dont les employeurs ne manqueront pas de tirer profit. Vers la fin du XIX ${ }^{e}$ siecle, les femmes et les enfants constitueront donc un élément important mais docile de la force de travail. Les femmes prendront alors graduellement conscience de leur statut d'infériorité et de la discrimination dont elles sont l'objet. De cette sensibilisation surgira un premier mouvement d'organisation militante à l'intérieur duquel sera recrutée la première génération de féministes.

5. Le concept du divorce n'est pas reconnu par la loi civile du Québec au $\mathrm{XIX}^{\mathfrak{e}}$ siècle. Le mariage n'est considéré dissoluble que lors de la mort naturelle d'un des deux conjoints. Le Code civil tolère cependant la « séparation de corps » qui permet aux conjoints de vivre sous des toits différents sans toutefois les autoriser à se remarier (Backhouse, 1991). 
La fin du $\mathrm{XIX}^{\mathrm{e}}$ siècle est également une période où le pays tout entier patauge dans un vaste courant visant à restaurer son intégrité sociale. En effet, les bouleversements socio-économiques importants qui agissent sur la société québécoise vers la fin du $\mathrm{XIX}^{\mathrm{e}}$ siècle $^{6}$ suscitent la montée d'un projet de société qui passera, entre autres, par l'amplification d'un discours sociétal cherchant à moraliser les mœurs - par le biais des campagnes de tempérance, par exemple - et à épurer les comportements sexuels des Canadiennes et des Canadiens. Outre les mours et la sexualité. ce nouveau discours moralisateur se fera particulièrement insistant à l'égard de la pauvreté ${ }^{7}$, des femmes et de la famille. Une peur croissante quant à la désintégration progressive du foyer familial -- modèle de reproduction de l'ordre social privilégié par la classe moyenne - va alors se fabriquer et incitera les réformateurs et les gouvernants à se pencher sérieusement sur la perpétuation de la famille patriarcale. Ce souci de préservation du noyau familial occasionne dès lors une prise en charge, par les moralistes, de la nature des femmes. Celles-ci en viennent à incarner la moralité et leur dérogation aux conventions leur vaudra souvent d'être reléguées à un statut de déchéance. Agissant vraisemblablement à titre de protecteur affable des intérêts de la classe moyenne qui cherche à assurer le maintien de l'ordre social, La Presse de cette époque proclame que l'émancipation des femmes ne constitue aucunement un progrès et qu'elles doivent demeurer l'âme dirigeante de la maison et l'ange gardien des valeurs familiales 8 .

L'emprise des moralistes sur le comportement des femmes à l'ère victorienne s'effectue sous une domination masculine de tous les aspects de la vie sociale, que ce soit dans l'Église ou dans la vie civile (prêtre, père, mari, législateur). À différentes époques de l'histoire, les hommes ont dû se définir une nouvelle société et ont attribué aux femmes une place particulière dans celle-ci. Or, dans le projet de société qui se dessine au tournant du $\mathrm{XX}^{\mathrm{e}}$ siècle, les hommes cantonneront les femmes dans une sphère où elles seront reines, le foyer domestique, et concéderont un rôle crucial à la

6. À la suite đ'une crise économique sérieuse qui bouleverse le monde en 1873 , le processus d'industrialisation de la province reprend son cours au début des années 1880. Néanmoins, en dépit des nouvelles industries foisonnantes, les conditions de vie des travailleurs et travailleuses demeurent précaires et engendrent le démarrage d'un syndicalisme structuré. De plus, la demande grandissante pour une main-d'œuvre à bon marché encouragera l'affluence de gens dans les villes et suscitera la prolifération des indigents et des marginaux. Les structures de contrôle et de service existantes à l'époque succombent sous la pression des populations et des problemes (Linteau et al., 1989; Rutherford, 1982).

7. Au XIXe siècle, la pauvreté est considerée comme étant un défaut individuel d'adaptation au système économique et social et la charité faite auprès des indigents n'est alors perçue que comme un privilège mérité par les nécessiteux qui n'ont pas sombré dans des habitudes honteuses comme l'intempérance (Linteau et al., 1989).

8. La Presse, 16 août 1888, article non signé, p. 2 . 
maternité qui constituera, dorénavant, la «normalité » féminine de l'époque (Collectif Clio, 1982). En somme, la fin du $\mathrm{XlX}^{\mathrm{e}}$ siècle est une période d'ébullition pour les femmes et de redéfinition de la place qu'elles occuperont à l'intérieur du projet social qui prend forme à ce moment. Or, c'est dans le cadre de ce contexte social qu'il devient intéressant de voir si les nouvelles prescriptions qui leur sont imposées se reflètent dans la construction journalistique qu'effectuera $L a$ Presse de l'image des femmes et en particulier, des femmes victimes de violence dans le foyer familial sacrosaint.

\section{II - VIOLENCE DOMESTIQUE : PRÉDOMINANCE DU « MARI BATTEUR 》}

La violence en ménage n'est pas un phénomène nouveau pour le Québec de la fin du XIX ${ }^{\mathrm{e}}$ siècle. Mais, ce qui est particulier à cette époque c'est que la «barbarie» de ce comportement devient sujet à débat public. notamment à partir des années 1870 . C'est dans cette période, en effet, que les voix des réformateurs sociaux de la classe moyenne s'unissent à celles des tenants des mouvements de tempérance dans le but de prémunir le public contre les effets pernicieux de l'abus d'alcool (Harvey, 1990). Les mouvements de tempérance, nombreux et solides à l'époque, contribuent largement à cette problématisation de la violence domestique puisque le problème des femmes battues y est invariablement associé à la consommation immodérée d'alcool. Une étude récente de Harvey (1991) souligne, à juste titre, le rôle primordial des mouvements de tempérance dans la dénonciation passionnée de la brutalité des maris aux prises avec l'alcool ainsi que dans la construction d'une attitude d'indignation du public face à la violence domestique. Par ailleurs, l'auteur fait également état de l'existence d'archives policières, judiciaires et journalistiques qui témoignent de la vindicte publique face à ce problème au Québec à l'époque.

Dans une orientation similaire à celle privilégiée par Harvey (1990, 1991), notre analyse se consacre à la perception journalistique des femmes victimes de violence domestique dans lc quotidien La Presse. Nos premières constatations suggèrent que la construction que le quotidien opère de l'image des femmes victimes de crimes présente de façon soutenue un discours sur la faiblesse de celles-ci. La fernme est dépeinte comme une pauvre victime qui subit invariablement un préjudice. On n'y retrouve en aucun cas une allusion à une provocation quelconque de la part de la femme. Cette constatation est surtout intéressante étant donné que cette même allusion prend une ampleur considérable tout au cours du $\mathrm{XX}^{\mathrm{e}}$ siècle. C'est, en effet, dans la première moitié du $\mathrm{XX}^{\mathrm{e}}$ siècle que les femmes se sont vu attribuer le blâme et la responsabilité de la situation de violence dans le ménage (Schur, 1983; Gordon, 1988). Mais il semble que vers la fin du siècle précédent ce ne fut pas encore le cas. 
L'idée générale qui se dégage de notre corpus veut que dans les délits que La Presse choisit de rapporter, la situation de la femme ne soit jamais discutée dans les textes journalistiques. L'image qui en est véhiculée doit être devinée et saisie principalement à travers la description du comportement de l'infracteur, c'est-à-dire du «mari batteur » ${ }^{9}$. Trois arguments principaux, issus de l'analyse, supportent cette première constatation.

Tout d'abord, la femme victime d'abus physiques est habituellement absente des constructions qu'effectue La Presse de ce type de délits; on ne parle tout simplement pas d'elle. Tant en ce qui a trait à la description de sa situation, à ses blessures ou même à son aspect physique, la femme est généralement laissée dans l'ombre par les journalistes sauf lorsqu'il s'agit minimalement de la plaindre :

...sa malheureuse femme est obligée de passer la journée dans une boutique... pendant que l'indigne mari s'enivre et dissipe une partie du salaire de sa malheureuse compagne. Il s'est rendu coupable d'un assaut brutal sur sa fernme... ${ }^{10}$

Cette femme [est] dans un état lamentable...[elle] fait pitié à voir. Elle est couverte de plaies et de contusions. ${ }^{11}$

Néanmoins, malgré le fait que le quotidien ne les présente pas ouvertement, nous pouvons dégager de ce type de narration une idée précise sur les femmes. Contrairement à celles qui subiront des sévices semblables au début du siècle suivant et qui se verront attribuer la responsabilité de leur situation de violence en ménage, les épouses du XIX ${ }^{\mathrm{e}}$ siècle sont plutôt présentées comme les victimes faibles, impuissantes et désemparées des «mauvais maris »: $: 12$

Pour assouvir sa rage, il se rua ensuite sur M. qu'il maltraita de la manière la plus barbare. La malheureuse est dans un état précaire et trop faible pour dénoncer le misérable qui l'a ainsi battue. ${ }^{13}$

... a tenté de tuer sa femme dans un accès de jalousie furieuse. La pauvre femme soutint la lutte avec un courage héroïque. ${ }^{14}$

9. La grande majorité des récits recensés désigne l'homme qui se porte à des actes de violence sur son épouse de « mari batteur ».

10. La Presse, 31 janvier 1889, p.4.

11. La Presse, 16 janvier 1889 , p. 4.

12. Le résultat de nos analyses ayant trait à cet aspect de la représentation des femmes dans La Presse est concordant avec les analyses effectués par Harvey (1991) a partir de textes journalistiques extraits du quotidien Montreal Star dans les annees 1869 à 1879.

13. La Presse 19 janvier 1886, p. 4.

14. La Presse, 31 mai 1886 , p. 3. 
Ce qui est ensuite particulier dans nos données, et qui ressort avec beaucoup d'insistance, c'est l'importance que prend le discours dénonciateur de la conduite masculine par rapport aux propos essentiellement ténus qui sont véhiculés sur les femmes victimes de ce type de comportements. En cette fin du $\mathrm{XIX}^{\mathrm{e}}$ siècle la notion du mari brutal devient intolérable et les raisons qu'il invoque pour justifier ses «manières rudes» sont jugées inadmissibles et font même régulièrement l'objet de commentaires sarcastiques de la part des journalistes :

... en temps d'ivresse, il trouve son épouse amère et le lien conjugal trop lourd... après une soûlographie en règle. [il] a battu sa femme hier. Le recorder lui a octroye $\$ 8.00 \ldots$ pour sa récompense. ${ }^{15}$

... l'homme accuse sa moitié d'être la créature la plus vicieuse du monde mais sa défense... est très mauvaise. ${ }^{16}$

V. prétend que la malice de son épouse est la cause de son ivrognerie. ${ }^{17}$

Cette tendance qu'a le journal à dénoncer de façon virulente le «mari barbare», «ivrogne et brutal », semble, dans une certaine mesure, conforme aux idéaux moraux des réformateurs de l'époque. On se rappelle que la réforme sociale, dont la classe moyenne de la seconde moitié du $\mathrm{XIX}^{\mathrm{e}}$ siecle se porte garante, propose en outre, de redresser la place de la famille au sein d'une nouvelle société morale. La volonté de moraliser les mours et de préserver la famille paraissait donc avoir mené à l'élaboration de normes de féminité et de maternité particulières. Or, il y eut, parallèlement aux premières, une diffusion de nouvelles prescriptions de masculinité et de virilité bourgeoises qui cherchaient, entre autres, à purifier le foyer familial de toute violence physique (Gardon, 1988). Ces prescriptions exilaient la contenance, le contrôle de soi et le règne paternel grâce à une autorité, certes, mais qui ne devait requérir aucune violence pour s'imposer. La violence exercée à l'égard de l'épouse était donc perçue comme une expression indigne de cette nouvelle orientation de l'« homme».

L'amplification de l'immoralité des comportements du mari laisse alors supposer que le quotidien participe à la construction et à la diffusion de la nouvelle image masculine promulguée dans cette période et qu'il condamne, par conséquent, toute pratique qui menace l'instauration du nouvel ordre social cher à la classe moyenne. La violence des maris paraît à ce moment-là prendre l'allure d'une pratique abjecte et peu honorable et ce, contrairement aux conceptions communes qui supposent qu'elle était un droit, sinon légitime du moins consenti au chef de famille. En réalité, nos

15. La Presse 18 février 1886, p.4.

16. La Presse, 14 avril 1886, p.4.

17. La Presse, 28 janvier 1886, p.4. 
résultats suggèrent que l'exercice d'un contrôle moral sur la famille — par la pacification du foyer - passe non seulement par la prise en charge de la nature des fernmes, mais inclut également la moralisation des comportements des hommes. Que ce soit à l'égard des « excès de boisson » ou de leur conduite envers les femmes, les hommes voient leurs comportements faire l'objet d'une certaine intention de «bourgeoisification". Ainsi, de manière parallèle à la normalisation de la conduite des femmes, le projet victorien cherchera vraisemblablement à «civiliser» celle des hommes en sanctionnant, entre autres, l'intempérance, l'absence de support familial ou, encore, la violence contre l'épouse.

Les délits rapportés par La Presse semblent, en effet, reconstruits de façon à mettre essentiellement l'accent sur l'atrocité du crime. Par le biais de la presse populaire. les réformateurs de l'époque feront donc de la violence domestique le véhicule idéal à travers lequel ressasser l'éternel combat entre le bien et le mal. L'image que le quotidien fabrique des protagonistes de ce type d'affaires passera alors par l'usage d'un code dichotomique qui présente de façon soutenue le mari comme le bourreau et l'épouse comme la suppliciée. Le vocabulaire privilégié par ce genre littéraire relève du mélodrame, mais il témoigne non seulement d'un goût du lecteur pour les sensations mais aussi, et surtout, d'un sentiment d'indignation de la part des journalistes quant à l'occurrence de ce type d'«acte odieux $»$ :

[le recorder] a séparé... un mari barbare... un monstre à la figure humaine, de son épouse qu'il maltraitait. La victime a perdu patience le matin du jour de l'an $1887 .$. . [où] il la roua de coups. Il a fait une martyre de sa Emma. Le Juge parla avec indignation de cette férocité révoltante... [de cet] exemple de sauvagerie. ${ }^{18}$

Il demanda de l'argent à sa femme qui était couchée. Sur son refus, il l'a [sic] sortit du lit et l'entraîna dehors en la battant et l'injuriant. Il s'élança pour donner une nouvelle dégelée à sa chère moitié. [II] se contenta de répondre... que ces femmes (mère et fille) exerçaient une vengeance contre lui et racontaient des mensonges. ${ }^{19}$

Enfin, lorsque le quotidien daigne présenter la victime - ce qui demeure très rare selon nos données - ce n'est qu'en termes du déshonneur dont elle est l'objet au moment de la dénonciation du délit :

À bout de force et de patience elle s'est enfin résignée à subir l'humiliation d'une arrestation, d'un procès et d'une sentence [de son mari]. ${ }^{20}$

18. La Presse, 12 janvier 1889 , p. 4.

19. La Presse, 9 mars 1886, p. 4.

20. La Presse 31 janvier 1889 , p. 4. 
En rendant leur insatisfaction publique, ces femmes ébranlent le modèle de la famille patriarcale et attaquent le mythe de la famille heureuse. Elles défient, de plus, les normes sociales qui condamnent la rupture du lien conjugal et encouragent la soumission et la passivité de la femme pieuse et vertueuse. Or, la préservation jalouse de tous ces éléments se retrouve au cour même du projet de réforme sociale qui est en pleine expansion au pays à l'époque. En fait, au XIX ${ }^{\mathrm{e}}$ siècle l'endurance des femmes a la victimisation qu'elles subissent de la part des « maris batteurs » caractérise davantage une vertu qu'une faiblesse et garantit leur crédibilité en tant que «bonne épouse». En effet, les femmes qui tolèrent l'abus semblent beaucoup plus louangées que celles qui dénoncent ou quittent leur mari. Harvey avait déjà souligné, en 1990, les hésitations des femmes à dénoncer leur mari et surtout à poursuivre les procédures judiciaires entamées par une mise en accusation. La reconstruction qu'opère ici La Presse de ces cas particuliers paraît effectivement miser sur la dénonciation comme l'ultime recours des femmes violentées. L'image de celles-ci en est alors une de la femme qui a accompli son devoir d'épouse en résistant le plus longtemps possible aux excès du mari :

Ce ménage est un véritable enfer. Tous les jours des scènes de pugilat se renouvellent mais... les choses ont dépassé les limites ordinaires, la femme ayant reçu à la tête une blessure grave, s'est décidée à faire arrêter son époux. ${ }^{21}$

Celle-ci a déclaré que depuis cinq ans elle est en butte aux persécutions de son mari. [Il] a proféré contre elle des menaces qui l'ont effrayée et elle $s^{\prime}$ est enfuie du toit conjugal. ${ }^{22}$

Madame C. souffrait depuis longtemps des mauvais traitements de son maraud de mari $!^{23}$

Ces extraits suggèrent qu'au tournant du siècle, la promotion moraliste de certaines vertus féminines comme l'obéissance et la complaisance, mais aussi la discipline, la responsabilité et la loyauté pouvait encourager la victimisation des femmes. Ainsi, en dépit du fait que le quotidien à l'étude reste sensiblement muet au sujet de la victime, il reste tout de même une certaine image de la femme qui se devine et se dégage de cette dernière analyse; celle de la pauvre épouse humiliée par le fait de se voir poussée au-delà des limites « raisonnables » — à l'intérieur desquelles la violence domestique est socialement tolérée - et de devoir dénoncer l'intransigeance de son mari. Cette image de l'épouse humiliée, véhiculée par $L a$

21. La Presse, 20 mars 1886, p. 4. Le souligné n'apparaît pas dans l'article original.

22. La Presse, 9 avril 1886, p. 4. Le souligné n'apparaît pas dans l'article original.

23. La Presse, 31 mai 1886, p. 3. 
Presse, est essentiellement tributaire des valeurs et des normes sociales promulguées, à l'époque, par la classe moyenne. Ainsi, la dénonciation virulente des «maris batteurs» est jugée indispensable à la légitimation des idées moralistes sur la redéfinition de l'homme et de la masculinité. Cependant, l'existence simultanée de critères nouveaux issus également de la classe moyenne, et concernant les attributs et les comportements féminins désormais appropriés (e.g. passivité, dépendance), condamne moralement les femmes qui y contreviennent en dénonçant aux autorités la violence du mari. Il semble donc que les restrictions morales, sociales ou légales imposées à l'époque à l'intempérance ou à l'endroit des maris violents aient eu comme principal objectif non pas la protection des femmes, mais le contrôle des comportements des hommes issus des milieux ouvriers, c'està-dire des milieux jugés de plus en plus dangereux de par leur instabilité et leur oisiveté (Frégier, 1840).

La présence notable des délits de violence domestique dans la presse écrite témoigne non seulement d'une propension sensationnaliste à les rapporter, mais surtout d'une tendance sociétale répressive. Cette constatation nous a, entre autres, permis de retenir un enseignement majeur de notre analyse. En effet, la conception des libertés qui est dominante dans le $\mathrm{XIX}^{\mathrm{e}}$ siècle en est une qui prône les droits individuels contre l'État. Or. cette mentalité participe à la prévalence de l'institution familiale de type patriarcal ainsi qu'à l'attribution de droits aux chefs de famille, ce qui fait en sorte que le foyer restera privé et inviolable. Ainsi, l'évocation des droits à la vie privée a-t-elle permis de supprimer la garantie publique de libertés pour certains individus, en l'occurrence les femmes. Le privé et le public étaient donc construits socialement, à l'époque, comme des sphères mutuellement exclusives. Puisque l'interaction entre mari et femme est perçue comme l'apanage du domaine privé, les autorités légales sont demeurées hésitantes à intervenir lors de disputes familiales; elles ont proclamé plutôt que «the sanctity of the family home pervades the world of law enfoncement...» (Martin. 1977 dans Schur, 1983, p. 162). Néanmoins, on ne peut plus penser que la violence au foyer faisait. à l'époque, l'objet d'une non-intervention quasi systématique. Harvey $(1990,1991)$ a récemment fait la lumière sur l'existence d'une prise en charge médiatique et judiciaire des affaires de violence domestique dans le dernier tiers du $\mathrm{XIX}^{\mathrm{e}}$ siècle à Montréal. Dans la même lignée, nos résultats suggèrent que certains aspects du privé étaient vraisemblablement étalés en public.

Tout d'abord, parce que la proportion des femmes violentées est considérable à l'intérieur du bassin de délits que La Presse choisit de rapporter et, ensuite, parce que le quotidien dénonce fébrilement le «mari batteur» en lui fabriquant une image amorale. Finalement, parce que d'entrée de jeu la 
période historique étudiée est réglée par une loi particulière portant sur les agressions contre les femmes ${ }^{24}$. Cet article de loi figure dans les statuts criminels de l'époque à titre d'infraction donnant lieu à une accusation libellée («indictable offense»), alors que les voies de fait simples ne constituent qu'une infraction donnant lieu à une condamnation sommaire. À la différence de la seconde catégorie - qui comprend les délits de moindre gravité jugés par un juge de paix ou un magistrat de police - les accusations libellées englobent les crimes «les plus atroces» et sont jugés par les tribunaux et jurys. Par ailleurs, les accusations libellées entraînent des peines d'incarcération potentiellement plus longues que les accusations sommaires qui. elles, conduisent le plus souvent à une amende quelquefois accompagnée d'une légère période de détention. Il appert que cette loi particulière a été rarement utilisée dans les cas de violence domestique. En effet, les accusations pour voies de fait contre les femmes passent, chez les hommes, de 29 en 1888 à 52 en 1899 et ce, pour l'ensemble du pays ${ }^{25}$. Mais notre analyse témoigne d'une inadéquation importante entre le nombre de condamnations pour ce type de délit et la pléthore des cas rapportés par $L a$ Presse.

Quelques raisons hypothétiques peuvent expliquer cet état de choses. Premièrement. il est plausible de supposer que les «maris batteurs» aient été, en général, accusés de voies de fait simples et ce, peut-être parce que les tribunaux jugeaient. à l'époque, que la peine prévue dans les cas de voies de fait contre les femmes était trop sévère et qu'ils estimaient que ce type de délit ne devait pas être réprimé si rigoureusement. Deuxièmement, il est possible que l'apparent laxisme de l'appareil judiciaire cache une autre pratique, moins rigoureuse mais tout de même répressive. En effet, la période étudiée se caractérise par la prolifération des campagnes de tempérance et la violence de l'homme y est perçue comme étant la conséquence directe de son vice dipsomane, d'autant plus que l'ivresse et l'ivrognerie étaient alors les euphémismes privilégiés pour atténuer la dure réalitê de la violence domestique (Harvey, 1991). Il est donc probable que les maris aient été plus souvent accusés d'ivresse et de tapage que de voies de fait contre leur épouse, ce qui contribuait à canaliser les plaintes vers des catégories d'infraction autres que celle comprenant les voies de fait contre les femmes. Mentionnons au passage que la crainte de représailles de la part du mari agissait fréquemment comme facteur de dissuasion important pour les épouses victimes. Cependant, il arrivait que les femmes portaient plainte contre leur mari mais devaient, dans ce cas, débourser des frais d'un dollar équivalant au coût du mandat d'arrestation (Harvey, 1990). Or, pour les femmes sans ressources financières, cette somme était considérable et a pu

24. Statuts révisés du Canada, 1886, 49 Vict, chap. 176, art. 3, alinéa D.

25. Canada, Annuaire statistique, 1899, p. 581-596. 
en rebuter plusieurs. Pour celles qui pouvaient défrayer le coût du mandat, l'arrestation du mari assurait son maintien derrière les barreaux jusqu'au début des procédures judiciaires, (ce qui garantissait, par le fait même, une certaine protection aux épouses). Pour plusieurs d'entre elles, cette incarcération suffisait puisque advenant le départ du mari du foyer familial pour un trop long séjour d'emprisonnement, les épouses faisaient face à un retrait obligé du support financier principal. Cela les confrontait à des possibilités restreintes; retirer tout simplement les doléances, n'accuser l'époux que d'assaut simple ou porter plutôt plainte pour non-support familial. Mentionnons que les plaintes des femmes à l'endroit des «maris batteurs» se faisaient d'usage par l'entremise des agences de protection de l'enfant. Toutefois, les situations de violence domestique semblaient être occultées en tant que problème par celles-ci. Alors, les doléances des femmes avaient plus de chances d'être considérées comme fondées si elles portaient sur des accusations de non-support familial plutôt que de violence directe sur l'épouse (Gordon, 1988).

\section{CONCLUSIONS}

Les résultats auxquels nous conduit notre l'analyse laissent plusieurs questions en suspens parmi lesquelles certaines peuvent s'avérer particulièrement riches. Il est probable que la dénonciation publique de la violence domestique, qui s'effectuait dans la presse écrite québécoise populaire à la fin du $\mathrm{XIX}^{\mathrm{e}}$ siècle, se retrouvait également au sein d'autres institutions sociales de l'époque. En tant que phénomène isolé, elle aurait été engendrée prioritairement par des considérations sensationnalistes de la part du quotidien La Presse. Par contre, une dénonciation à plus grande échelle, de la part de la classe moyenne et des tenants des mouvements de tempérance. replace la violence contre les épouses au cceur d'une conscientisation sociétale générale contre la violence au foyer qui, à travers des décisions politiques et sociales, se concrétise dans un contrôle plus accru et plus serré d'un comportement désormais jugé répréhensible. Initialement, La Presse est un organe de propagation d'idées conservatrices ${ }^{26}$ ce qui la rend favorable aux intérêts de la classe moyenne, à ses débuts du moins. Sans laisser entendre que le quotidien est à la remorque d'intérêts sociaux précis, nous pouvons toutefois supposer qu'il fonde ses interventions sur une vision particulière de l'ordre social et qu'il a, dès comme lors, tendance à dénoncer toute

26. L'avènement de La Presse est le résultat d'une scission au sein du parti conservateur fédéral qui, dans les années 1880 , entraîne sa division en deux factions. C'est principalement face à la nécessité pour ces factions de compter sur un organe de presse que fut créé, en 1884, le quotidien La Presse. Malgré son caractère éventuellement populiste, La Presse demeure, à ses débuts, favorable aux intérêts conservateurs (Felteau, 1983). 
pratique considérée comme une menace à cet ordre. À ce titre, les pratiques journalistiques privilégiées par La Presse parviennent à imposer une représentation particulière de la violence domestique et jouent donc un rôle clé dans le processus de problématisation sociale du phénomène. Les questions qui demeurent ouvertes concernent, dès lors, les autres institutions participant à cette construction de la violence en ménage comme problème social à la fin du XIX ${ }^{\mathrm{e}}$ siècle. Quelles étaient-elles? Et quelle était l'envergure moraliste et la portée disciplinaire d'une telle problématisation de la violence domestique?

Les récits journalistiques recensés au cours de cette étude indiquent que la «violence conjugale» n'était pas nommée comme telle au tournant du $\mathrm{XX}^{\mathrm{e}}$ siècle mais qu'elle faisait plutôt l'objet d'une appellation autre. Il reste à supposer, par conséquent, que ce concept a été ultérieurement fabriqué à des fins particulières et que certains éléments conjoncturels du contexte socio-politique ont été mis à contribution dans la construction de la nouvelle catégorie sociale de comportements que deviendra plus tard la "violence conjugale». Par conséquent, la question de l'évolution même de la notion, autant à l'intérieur du discours que dans les milieux d'intervention. demeure tout entière.

Par ailleurs, les représentations sociales ne constituent cependant pas l'essentiel de la création notionnelle puisque la «violence conjugale» se traduira concrètement dans un traitement social particulier des individus dont les comportements seront affublés de la nouvelle étiquette. À cet égard on en est venu, au $\mathrm{XX}^{\mathrm{e}}$ siècle, à attribuer le blâme de la situation de violence à la femme qui en est victime et à la traiter comme responsable (Lévesque. 1989). Mais puisque nos résultats nous signalent que l'attribution du blâme ne faisait pas encore partie de la représentation que la presse populaire entretenait à l'égard des femmes victimes de violence domestique, ils soutiennent, par conséquent, l'idée que la construction de l'image sociale de la «femme battue » a fait indubitablement l'objet d'une évolution quant aux critères la définissant comme telle. Il reste donc à retracer au cours du $\mathrm{XX}^{e}$ siècle des points tournants marquants dans cette évolution et a vérifier comment, à travers ceux-ci, la «femme battue» en viendra à prendre la place qui lui sera attribuée où elle sera dépeinte comme la source de tous les torts.

La lumière qu'une étude de ce genre permet de faire sur la représentation qu'entretenait une des institutions de la société québécoise de la fin du siècle dernier sur les femmes victimes de violence au foyer, encourage, par ailleurs, la formulation de plusieurs pistes éventuelles de réflexion. Au-delà du rôle reconnu des mouvements de tempérance dans la problématisation de la violence domestique au siðcle passé (Gordon, 1988; Harvey, 1990, 1991 ; Bradbury, 1993), une analyse doit aussi être attentive aux représentations 
qui légitiment l'intervention des defenseurs de la sobriêté. L'intêrêt pour les représentations nous permettrait de mieux saisir d'où les mouvements de tempérance tirent leur discours et leurs pratiques. L'analyse peut même aller plus loin en accordant une importance à d'autres représentations qui pourraient éventuellement participer au processus de construction d'un problème social (e.g. syndicats, Église). En réalité, cet article ouvre la porte à une réflexion beaucoup plus large qui permet de repenser la dynamique victorienne dans son ensemble. Il est généralement admis que le projet d'incapacitation des femmes est une vérité transhistorique qui demeure relativement invariable. On a alors pensé que les aspects qui gravitent autour de cette intention sont constants par ailleurs et que la normalisation ne vise que les femmes. Or, nos résultats suggèrent que ce n'était pas le cas dans le projet victorien du $\mathrm{XIX}^{\mathrm{e}}$ siècle et que la conduite des hommes faisait aussi l'objet d'un discours moralisateur et ce, au même titre que la pauvreté, la famille et les mours des femmes.

\section{BIBLIOGRAPHIE}

\section{Sources imprimées}

Canada, Annuaire statistique, 1899, p. 581-596.

La Presse, janvier 1886 à décembre 1889.

Statuts révisés du Canada, 1886, 49 Vict., chat. 176, art. 3, alinéa D.

\section{Travaux contemporains}

BACKHOUSE, (1991), Petticoats and Prejudice: Women and Law in NineteenthCentury Canada, Toronto The Osgoode Society.

BEAULIEU, A., HAMELIN, J. (1977), La Presse québécoise des origines d nos jours. Tome III (1880-1895), Quéhec, Les Presses de l'Université Laval.

BRADBURY B. (1993), Working Families : Age, Gender and Daily Survival in Industrializing Montreal, Toronto, McClelland \& Stewart.

COLLECTIF CLIO (1982), L'histoire des femmes au Québec depuis quatre siècles, Montréal, Quinze.

CROSS, S. (1973), « The Neglected Majority: The Changing Role of Women in luth Century Montreal », Histoire sociale, vol. $6, \mathrm{n}^{\circ} 12 ;$ p. 202-224.

CURRAN, J. (1978), "The Press as an Agency of Social Control : an Historical Perspective», in G. Boyce et al. New spaper History : from the Nineteenth Century to the Present Day, London, Constable, p. 51-75.

DUMONT-JOHNSON, M., «Histoire et condition de la femme dans la province de Québec ", Tradition, culture et histoire politique de la femme au Canada, étude $\mathrm{n}^{\circ} 8$ (Ottawa, Commission royale d'enquête sur la situation de la fermme au Canada, 1971), 59 p. 
ERICSON, R. (1987), Visualizing Deviance, Toronto, University of Toronto Press.

FELTEAU, C. (1983), Histoire de La Presse. Tome I. Le livre du peuple. 1884.1916, Montréal, Les Éditions La Presse.

FRÉGIER, H.-A. (1840), Des classes dangereuses de la population dans les grandes villes et des moyens de les rendre meilleurs [sic], Bruxelles, Meline.

GORDON, L. (1988) Heres of their own lives: The Politics and History of Family Violence, Boston 1880-1960, New York, Viking.

HARVEY, K. (1990), « To love, Honour and Obey: Wife-battering in Working-Class Montreal, 1869-79 ", Revue d'histoire urbaine, vol. 19 (2) p. 128-140.

HARVEY, K. (1991), "Amazons and Victims : Resisting Wife-Abuse in WorkingClass Montreal, 1869-79», Revue de la Société historique du Canada, nouvelle série, vol. 2 p. 131-148.

LÉVESQUE, A. (1989), La norme et les déviantes: des femmes au Québec pendant l'entre-deux guerre, Montréal, Éditions du Remue Ménage.

LINTEAU, P.-A. et al. (1989), Histoire du Québec contemporain : De la Confédération à la crise (1867-1929), Montréal, Boréal.

RUTHERFORD, P. (1982), A Victorian Authority: The Daily Press in Late NineteenthCentury Canada, Toronto, University of Toronto Press.

SCHUR, E. (1983), Labeling Women Deviant: Gender. Stigma and Social Control, Philadelphie, Temple University Press.

SNELLL, J.G. (1983), «The White Life for Two " : The Defence of Marriage and Sexual Morality in Canada. 1890-1914», Histoire sociale, vol. 16, $\mathrm{n}^{\circ} 31, \mathrm{p} .111-130$.

VOUMVAKIS, S., ERICSON, R. (1984), News Accounts of Attacks on Women: A Comparison of Three Toronto Newspapers, Toronto, Centre of Criminology, University of Toronto. 